\title{
A Simple Emotion Discrimination Technique Based on Triangle Phase Space Mapping of HRV Signals
}

\author{
Ateke Goshvarpour ${ }^{\mathrm{a}}$, Ataollah Abbasi ${ }^{\mathrm{a}, *}$, Atefeh Goshvarpour ${ }^{\mathrm{a}}$ \\ ${ }^{a}$ Department of Biomedical Engineering, Faculty of Electrical Engineering, Sahand University of Technology, \\ Tabriz, Iran
}

\begin{abstract}
Physiological signal processing techniques are commonly used in emotion recognition. Heart rate variability (HRV) is an important tool in disease diagnosis and psychological investigations. Because of the chaotic nature of HRV, customary methods may not be proficient. Taking the advantage of geometrically based algorithms can lead to the uncomplicated and better representation of heart rate dynamics. The aim of this study was to test whether a simple HRV measure, based on triangle phase space mapping and polynomial fitting, provides a useful emotion recognition technique. HRV of women $(n=12)$ aged 19-25 years were compared to that of 12 matched aged men, while subjects were induced by four emotional stimuli: happy, sad, afraid, and relax. Kruskal-Wallis test was applied to show the level of significance of the features. The results confirm that emotional responses to sad, afraid and relax stimuli can be differentiated by the proposed indices. In addition, they are significantly different in both genders' physiological reactions. It seems that the suggested simple quantifiers are most promising in offering new insight into the dynamics assessments of HRV signals in different emotional states.
\end{abstract}

Index Terms: Emotion, Gender, Heart Rate, Nonlinear Dynamics, Statistical Data Analysis.

(C) 2017 Published by MECS Publisher. Selection and/or peer review under responsibility of the Research Association of Modern Education and Computer Science.

\begin{tabular}{|ll}
\hline \multicolumn{2}{l}{ Nomenclature } \\
ANS & Autonomic Nervous System \\
HR & Heart Rate \\
HRV & Heart Rate Variability \\
ECG & Electrocardiogram \\
IAPS & International Affective Picture System \\
\hline
\end{tabular}

* Corresponding author. Tel.: +98 4133459356; fax: +98 4133444322

E-mail address: ata.abbasi@sut.ac.ir 


\section{Introduction}

Autonomic nervous system (ANS) activity is considered as a main and important response in emotion. With the interpretation of the cardiovascular responses, quite a lot of information about autonomic functioning can be obtained. In research conducted in the field of emotion, different cardiovascular measures have been evaluated. Most studies focus on heart rate (HR) and heart rate variability (HRV) as a quantified physiological variable (Kreibig, 2010). HRV is a measure of cardiovascular control utilized by both sympathetic and parasympathetic nervous systems.

Generally, HRV examination has been performed using linear methods such as spectral analysis (Task Force, 1996). However, some studies have indicated there are more complex patterns in the signals that represent nonlinearity in the processes (Glass, 1999). Therefore, a linear approach such as power spectrum is not sufficient in HRV assessment and instead some nonlinear techniques have been proposed. Poincare plot is commonly used for nonlinear analysis of HRV, where the correlation between successive points of a signal with a geometrical illustration is offered.

Due to the simplicity of mathematical formulation and providing an indication of short-term and long-term HRV, a variety of quantitative methods have been presented. The ellipse-fitting technique is usually adopted by researchers (Tulppo et al., 1996), where fitting of an ellipse in the plot shape, the width and the length of the plot is measured through the dispersion of the points along minor (SD1) and the major (SD2) axis, respectively. SD1 and SD2, the measures of lag-1 correlation, represent the short-term and long-term fluctuations of the $\mathrm{HRV}$, respectively. Later, multiple lag correlation is also introduced to analyze the behavior of the signal (Goshvarpour et al., 2011).

Recently, some researchers have emphasized on the limitations of these analytical methods. They are linear, not being able to show spontaneous nonlinear fluctuations of the signal.

To overcome this problem, some new approaches are proposed such as triangular mapping phase space (Moharreri et al., 2014), where a second-order polynomial equation was offered to quantify the phase space. The capability of the proposed technique to discriminate different arrhythmia from normal sinus rhythm is established. In the current study, it is aimed that modified features of the mapping introduced and the capabilities of these features examined in the problem of emotion recognition through different genders.

The rest of this research is organized as follows. Section 2 provides the material and methods used in this work. The experimental results of the proposed algorithms are reported in section 3. Finally, conclusions are offered.

\section{Related Works}

Considerable efforts have been made to provide a reliable system for the emotion recognition. Since different modalities involved in emotions, several paradigms have been evaluated in automatic affect recognition. The two most important approaches are based on facial (Inam et al., 2015) and physiological parameters (Goshvarpour et al., 2016). However, the advantage of physiological signal analysis is that they cannot be simply faked by the participant. Therefore, biomedical data processing attracted the attention of several researchers.

Earlier, different methodologies have been employed using bio-signals. The first attempts benefited from linear based time-series processing (Kim et al., 2004). Because of simplicity and easy implementation, these methods are still frequently applied. However, due to the chaotic nature of biological systems, nonlinear based techniques have been proposed (Goshvarpour et al., 2016). Among several nonlinear approaches, graphical representation and trajectory based techniques are the popular ones. Consequently, this study adopted a geometrical-based analysis using the triangular mapping phase space.

Among physiological signals, cardiac functions play a major role in emotion. The review of 134 publications in emotion (Kreibig, 2010) revealed that among heart parameters, HR and HRV are two cardiac variables that most often reported in the literature. Therefore, in the current study, HRV analysis has been selected. 
On the other hand, individual differences have an influence on emotion responses (Fischer et al., 2004; Kunzmann and Grühn, 2005; Codispoti et al., 2008). The gender difference is an individual characteristic, which frequently observed in the emotion literature (Codispoti et al., 2008; Jönsson and Sonnby-Borgström, 2003). It prompted the authors of this study to consider gender differences in the results.

\section{Materials and Methods}

To perform this study, the following steps have been adopted. First, ECG signals were acquired when the subjects watching affective visual stimuli. Second, the signal was segmented based on the duration of emotional blocks. Third, the HRV was calculated. Fourth, the triangle phase space mapping was implemented to the polynomial coefficients were estimated. Next, the statistical Kruskal-Wallis test was performed. Finally, the role of gender differences was evaluated.

\subsection{Data Collection}

To understand the physiological fluctuations elicited by emotional images, electrocardiogram (ECG) of 24 college students attending Sahand University of Technology, 12 females (mean age: $21.75 \pm 1.86$ years) and 12 matched aged males (mean age: $21.25 \pm 1.42$ years) were collected. All participants were Iranian students.

To elicit emotions in subjects, images from the International Affective Picture System (IAPS) were applied (Lang et al., 2005). The IAPS comprises about hundreds of images which are emotionally evocative. The dataset is assessed by some American participants on three dimensions (on a discrete 9-point scale): valence, arousal, and dominance. In addition, participants also rated the felt emotion using an emotion wheel. For all dimensions, the mean and variance of participant assessments were calculated from these evaluation scores (Lang et al., 2005).

The images of the IAPS which are corresponding to four classes of emotions were chosen: relax, happy, sad and afraid (Goshvarpour et al., 2015). For each class of emotion, 35 images per category (the total of 140 images) were selected, varying on arousal (relaxing/stimulating) and valence (pleasant/unpleasant).

Upon arrival at the laboratory, all participants were asked to read and sign a consent form, which they only signed if they agreed to take part in the experiment. The participants were asked to determine if there were any particular circumstances in their life at that moment, which would cause them to be in a very aroused or very relaxed state. All participants reported no history of neurological disease. In addition, in a brief preliminary questionnaire, none reported cardiovascular, epileptic, and hypertension diseases. The subjects were asked not to use caffeine, fat or salty foods two hours before data recording. The mean temperature of the room was about $25^{\circ} \mathrm{C}$.

The participants were instructed to try and remain still during data recordings, particularly avoidance of hand, finger and leg movements. During the experimental procedure, the subject seated in front of the laptop screen (15.5-inch VAIO E Series) and his/her physiological responses (ECG / lead I) recorded. As shown in Fig. 1, the whole procedure was about 15 minutes and images were represented after 2 minutes of the initial baseline measurement in which subjects were instructed to keep their eyes open and watching a blank screen. Then, they watched the images while being aware of their emotional states. 28 blocks of pictorial stimuli were brought to the screen in a random order to avoid habituation in subjects. However, they were similarly balanced among subjects. Each block consists of 5 pictures from the same emotional class displayed for about 15 seconds with a 10 second of the blank screen period at the end of each block. This process was done to ensure the stability of the emotion over time. The blank screen period was applied to allow the physiological fluctuations to return to the baseline values and ensure regularity in the demonstration of different emotional images. The blank screen is followed by a white plus (for 3 seconds) to prompt the subjects to concentrate and look at the center of the screen and to prepare them for the next block. Fig. 1 demonstrates the protocol description. 


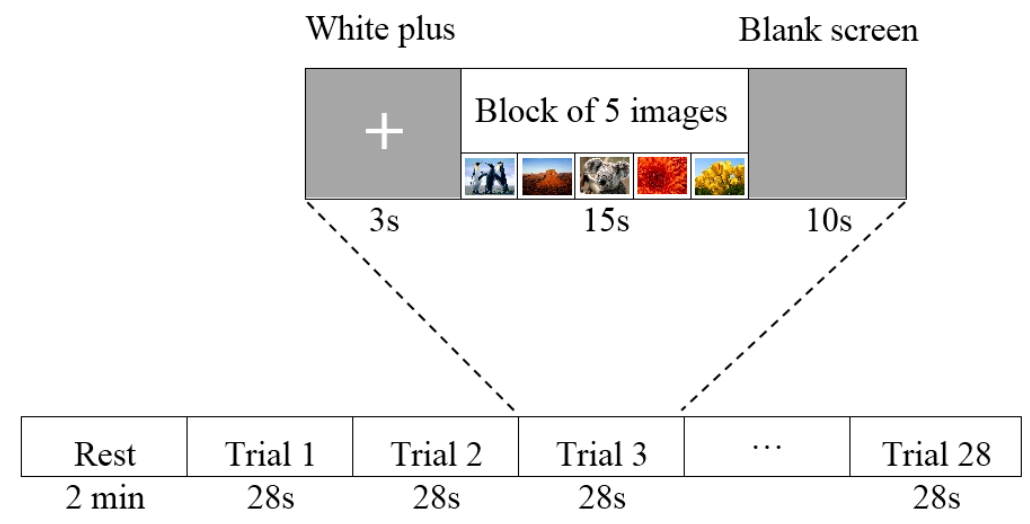

Fig.1. Protocol Description

As emotions are dependent on the past experiences of the subjects, they were asked to self-assess their emotional states.

All signals were recorded in Computational Neuroscience Laboratory of Sahand University of Technology using 16-channel PowerLab (manufactured by ADInstruments) with a sampling rate of $400 \mathrm{~Hz}$. A digital notch filter was applied to the data at $50 \mathrm{~Hz}$ to remove any artifacts caused by alternating current line noise. Finally, the HRV was extracted from the ECG.

\subsection{Triangle phase space mapping}

Considering the RR time series, the conventional Poincare plot has two dimensions and a lag of 1 interval, each point on the coordinate plane consists of a pair of consecutive intervals $\left(R_{\mathrm{i}}, \mathrm{RR}_{\mathrm{i}+1}\right)$. Where, $i=1,2$, $3, \ldots, n$ and $n+1$ is the length of the RR time series.

Instead, in triangle phase space mapping (Moharreri et al., 2014) the ordered pairs are formed as follows:

$$
\begin{aligned}
& \left(R R_{i}, y_{i}\right) \\
& y_{i}=\left(R R-R R_{i}\right)^{2}, \quad R R=\frac{1}{n+1} \sum_{i=1}^{n+1} R R_{i}
\end{aligned}
$$

Based on the distribution points, a polynomial was fitted to it. Unlike the previous study (Moharreri et al., 2014) in which a two-order polynomial was used, the four degrees polynomial was estimated in form of:

$$
Y=A X^{4}+B X^{3}+C X^{2}+D X+E
$$

where the polynomial coefficients (i.e. A, B, C, D, and E) construct the feature vector.

\subsection{Statistical analysis}

In the current study, the Kruskal-Wallis test is performed, which is a nonparametric version of the classical one-way ANOVA, and an extension of the Wilcoxon rank sum test to more than two groups. In this test, the null hypothesis that the features in two vectors are independent samples from identical continuous distributions with equal medians, against the alternative that they do not have equal medians is considered. 


\section{Results}

The triangle phase space mapping was constructed for four different emotional conditions: relax, happy, sad, and afraid. Fig. 2 exemplifies a sample.

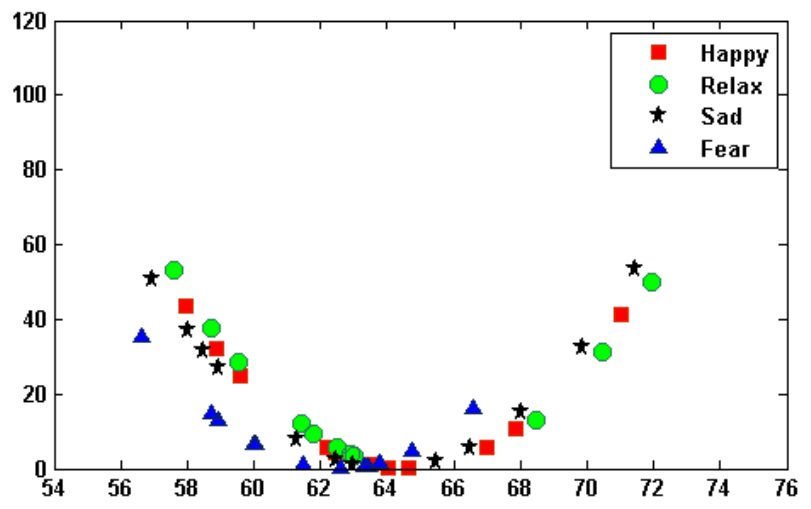

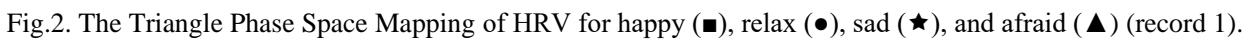

Comparing the plots, different morphologies of the estimated curves can be recognized. Therefore, it can be concluded that HRV signals have different behaviors during different affective states. For instance, the distribution, centroid, start, and the end of points are different in four states. To quantify these differences, the polynomial coefficients were extracted and the statistical test was performed to examine the significance of the features. The results of the statistical test for each feature are presented in Table 1.

Table 1. Statistical Results for Polynomial Coefficients

\begin{tabular}{|c|c|c|c|c|c|c|}
\hline \multirow[t]{2}{*}{ Affective states } & \multirow[t]{2}{*}{ Gender } & \multicolumn{5}{|c|}{ Polynomial Coefficients } \\
\hline & & $\bar{A}$ & $\mathrm{~B}$ & $\bar{C}$ & $\mathrm{D}$ & $\bar{E}$ \\
\hline \multirow[t]{2}{*}{ Sad vs. Relax } & women & $0.0039^{*}$ & $0.0039 *$ & $0.0039 *$ & 0.2480 & 0.2985 \\
\hline & men & $0.0039^{*}$ & $0.0039 *$ & $0.0039 *$ & 0.6032 & 0.6032 \\
\hline \multirow[t]{2}{*}{ Happy vs. Relax } & women & $0.0039 *$ & $0.0039 *$ & $0.0039 *$ & 0.8624 & 0.9080 \\
\hline & men & $0.0209^{*}$ & 0.0939 & $0.0326^{*}$ & 0.9539 & 0.9539 \\
\hline \multirow[t]{2}{*}{ Afraid vs. Relax } & women & $0.0039^{*}$ & $0.0039 *$ & $0.0039 *$ & 0.1840 & 0.2251 \\
\hline & men & $0.0039 *$ & $0.0039 *$ & $0.0039 *$ & 0.7289 & 0.7289 \\
\hline \multirow[t]{2}{*}{ Sad vs. Happy } & women & $0.0110^{*}$ & 0.0646 & 0.0734 & 0.2038 & 0.3261 \\
\hline & men & $0.0039^{*}$ & $0.0039 *$ & $0.0039 *$ & 0.6860 & 0.6032 \\
\hline \multirow[t]{2}{*}{ Afraid vs. Happy } & women & $0.0179 *$ & $0.0179 *$ & $0.0179 *$ & 0.2038 & 0.2251 \\
\hline & men & $0.0039 *$ & $0.0047 *$ & $0.0079 *$ & 0.7727 & 0.7727 \\
\hline \multirow[t]{2}{*}{ Sad vs. Afraid } & women & $0.0047^{*}$ & $0.0047 *$ & $0.0130 *$ & 0.8173 & 0.8173 \\
\hline & men & 0.4882 & $0.0243^{*}$ & $0.0209 *$ & 0.8624 & 0.8624 \\
\hline
\end{tabular}

Note- $*: p<0.05$ 
Applying the Kruskal-Wallis test (Table 1), it has shown that there is a significant difference between each pair of emotional states for coefficients of A, B, and C in men and women ( $p<0.05)$. In other words, not only the proposed parameters were significantly different in both genders, they also unbiased by the type of paired emotions to be discriminating. They differentiate sad, happy, and afraid from relax state; sad and afraid from happy state; and sad from afraid state, considerably. However, the smallest $p$-values assigned into the differentiation between relax and other affective states in both groups of men and women. More precisely, sad, happy, and afraid shows significant differences from relaxing state. It should be borne in mind that happy were also discriminated from afraid and sad with small $p$-values in men's groups. Therefore, it can be stated that significant differences between emotional responses were higher in men.

To examine whether there are any contrasts between men's and women's emotional reactions, gender difference is also evaluated (Table 2).

The results show that the coefficients of $\mathrm{A}, \mathrm{B}$, and $\mathrm{C}$ are influenced by the gender in all emotions except for happiness. In other words, men and women express dissimilar autonomic indices during afraid, sad and relax states.

Table 2. Statistical Differences between Men and Women for Polynomial Coefficients

\begin{tabular}{|c|c|c|c|c|c|}
\hline \multirow[t]{2}{*}{ Affective state } & \multicolumn{5}{|c|}{ Polynomial Coefficients } \\
\hline & A & B & $\mathrm{C}$ & $\mathrm{D}$ & $\mathrm{E}$ \\
\hline Afraid & $0.0039 *$ & $0.0039 *$ & $0.0047 *$ & 0.9080 & 0.8173 \\
\hline Sad & $0.0039 *$ & $0.0039 *$ & $0.0039 *$ & 0.7289 & 0.6860 \\
\hline Happy & 0.2251 & 0.3261 & 0.7727 & 0.3554 & 0.4527 \\
\hline Relax & $0.0039 *$ & $0.0039 *$ & $0.0039 *$ & 0.4187 & 0.4882 \\
\hline
\end{tabular}

Note- $*: p<0.05$

\section{Discussion and Conclusions}

In the current study, it is attempting to answer the following assumptions: 1) the potential of the proposed features (based on triangle phase space mapping and fitted polynomial coefficients) for studying emotions. 2) Whether there are any differences between men's and women's affective states. 3) The effect of different emotions (with different arousal and valence scores) on cardiac function of men against women. To this end, the polynomial coefficients fitted on triangle phase space mapping were extracted.

The results indicate that the proposed technique is useful for differentiating between men's and women's emotional responses to sad, afraid and relax inducements. However, there are not any significant changes for men's in contradiction of women's autonomic responses during happy state (Table 2). In addition, significant differences between each pair of emotional states (Table 1) for coefficients of A, B, and C are observed $(p<$ $0.05)$.

Previously, to characterize the chaotic time series trajectories, some geometric indices have been proposed (Goshvarpour et al., 2011; Moharreri et al., 2014; Yang, 2005). Useful information for nonlinear signal classification is attained by means of the geometric configurations of Poincare trajectories in terms of computer vision (Yang, 2005). For distinguishing different arrhythmia, Moharreri et al. (2014) evaluated a phase space mapping technique of HR. They found the usefulness of the features in arrhythmia discrimination. Since the proposed features have not been considered in the previous studies of emotion, it seems that these indices can be useful in differentiating between affective states and gender impacts.

There may be some considerations for improvement in the future studies: more data should be tested to generalize the proposed framework. Further investigations on different emotional categories are also in need.

Finally, with the examination of classification approaches, the implementation of the features is precisely scrutinized. 


\section{Acknowledgements}

The authors sincerely acknowledge Computational Neuroscience Laboratory, where the data were collected and all the subjects volunteered for the study.

\section{References}

[1] Kreibig SD. Autonomic nervous system activity in emotion: A review. Biol Psychol 2010;84:394-421

[2] Task Force of the European Society of Cardiology and the North American Society of Pacing and Electrophysiology. Heart rate variability: Standards of measurement, physiological interpretation and clinical use. Circulation 1996;93(5):1043-65

[3] Glass L. Chaos and heart rate variability. J Cardiovasc Electrophysiol 1999;10:1358-60.

[4] Tulppo M, Makikallio T, Takala T, Seppanen T, Huikuri H. Quantitative beat-to-beat analysis of heart rate dynamics during exercise. Am J Physiol 1996;271:H244-52.

[5] Goshvarpour A, Goshvarpour A, Rahati S. Analysis of lagged Poincare plots in heart rate signals during meditation. Digit Signal Process 2011;21:208-14.

[6] Moharreri S, Rezaei S, Dabanloo N, Parvaneh S. Extended parabolic phase space mapping (EPPSM): Novel quadratic function for representation of heart rate variability signal. Comput Cardiol 2014;41:417-20.

[7] Inam H, Malik A, Hayat M, Ashraf A. A survey on facial expression recognition technology and its use in virtual systems. I. J. Engineering and Manufacturing 2015; 2: 11-8.

[8] Goshvarpour A, Abbasi A, Goshvarpour A. Dynamical Analysis of Emotional States from Electroencephalogram Signals. Biomed Eng-App Bas C 2016; 28(2): 1650015.

[9] Kim K, Bang S, Kim S. Emotion recognition system using short-term monitoring of physiological signals. Med Biol Eng Comput 2004; 42(3): 419-27.

[10] Fischer AH, Rodriguez Mosquera PM, van Vianen AE, Manstead AS. Gender and culture differences in emotion. Emotion 2004; 4(1): 87-94.

[11] Kunzmann U, Grühn D. Age differences in emotional reactivity: the sample case of sadness. Psychol Aging 2005; 20(1): 47-59.

[12] Codispoti M, Surcinelli P, Baldaro B. Watching emotional movies: affective reactions and gender differences. Int J Psychophysiol 2008; 69(2): 90-5.

[13] Jönsson P, Sonnby-Borgström M. The effects of pictures of emotional faces on tonic and phasic autonomic cardiac control in women and men. Biol Psychol 2003; 62(2): 157-73.

[14] Lang P, Bradley M, Cuthbert B. International affective picture system (IAPS): Affective ratings of pictures and instruction manual. University of Florida, Gainesville, FL; 2005.

[15] Goshvarpour A, Abbasi A, Goshvarpour A, Affective visual stimuli: Characterization of the picture sequences impacts by means of nonlinear approaches. Basic Clin Neurosci 2015;6:209-221.

[16] Yang S. Nonlinear signal classification in the framework of high-dimensional shape analysis in reconstructed state space. IEEE Trans Circuits Syst II Express Briefs 2005;52:512-6. 


\section{Authors' Profiles}

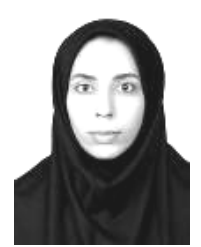

Ateke Goshvarpour received the M.Sc. in Biomedical Engineering from Islamic Azad University, Mashhad Branch, Iran in 2010. Since 2012, she is a Ph.D. student at Sahand University of Technology, Tabriz, Iran. Her research interests include biomedical signal processing, emotion recognition, neuroscience, nonlinear analysis, neural networks, and mathematical modelling.

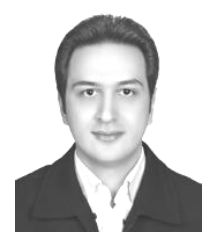

Ataollah Abbasi received the B.Sc. degree in biomedical engineering from Sahand University, Tabriz, in 2003, the M.Sc. degree in biomedical engineering from Sharif University of technology, Tehran, in 2005, and the Ph.D. degree in biomedical engineering from Sharif University of technology, Tehran, in 2010. Currently, he is a faculty member (associate professor) at Sahand University of technology in Tabriz, Iran. His research interests include Neuroscience (biomedical signal processing and modelling of neurological and mental disorders) and Cognitive Science (mental workload, creativity, emotion recognition, music therapy).

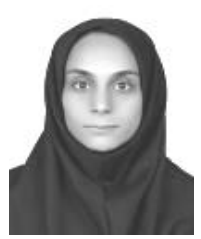

Atefeh Goshvarpour received the M.Sc. in Biomedical Engineering from Islamic Azad University, Mashhad Branch, Iran in 2010. Since 2012, she is a Ph.D. student at Sahand University of Technology, Tabriz, Iran. Her research interests include biomedical signal processing, emotion recognition, neuroscience, nonlinear analysis, neural networks, and mathematical modelling.

How to cite this paper: Ateke Goshvarpour, Ataollah Abbasi, Atefeh Goshvarpour,"A Simple Emotion Discrimination Technique Based on Triangle Phase Space Mapping of HRV Signals", International Journal of Engineering and Manufacturing(IJEM), Vol.7, No.3, pp.41-48, 2017.DOI: 10.5815/ijem.2017.03.05 